\title{
Cyclic Magnetic Actuation Technique for Thin Film Interfacial Fatigue Crack Propagation
}

Gregory T Ostrowicki* and Suresh K Sitaraman

George W. Woodruff School of Mechanical Engineering, Georgia Institute of Technology, 813

Ferst Drive, Atlanta, GA 30332

gtostrowicki@gatech.edu, suresh.sitaraman@me.gatech.edu

*Corresponding author. Present address: 2061 Glencoe Dr, Rockwall, TX 75087

\begin{abstract}
Interfacial fatigue crack characterization is challenging for as-deposited thin films due to issues with fixturing and precision application of cyclic loading. A fixtureless and microfabricatedtest technique is presented to characterize delamination propagation rate as a function of constant amplitude fatigue loading using a novel peel test configuration and magnetic actuation. The test was demonstrated for $1.6 \mu \mathrm{m}$ thick $\mathrm{Cu}$ films on a Si substrate, where interfacial fatigue crack propagation was observed to follow Paris Law behavior.
\end{abstract}

Keywords: $\mathrm{Cu}$ thin film, interfacial delamination propagation, cyclic fatigue, magnetic actuation

\section{INTRODUCTION}

Thin film delamination is often responsible for device failure, and is a major reliability concern in applications such as microelectronics, solar cells, flexible displays, microelectromechanical systems (MEMS), and many others. For example, sudden catastrophic delamination can occur due an unforgiving fabrication process or an overload in the field.

Interfacial cracks can also nucleate and grow incrementally over many subcritical loading cycles, causing premature device failure. Current experimental test techniques are mainly suited to characterize thin film interfacial fracture toughness under monotonic loading. In other words, these techniques determine the critical load at which fracture occurs, however they are generally ill-suited to provide rapid and precise cyclic loading for a fatigue crack propagation (FCP) study. Consequently, there is a general lack of data in the literature concerning interfacial delamination under cyclic fatigue loading for virtually all thin film material systems. 
For characterization of thin film adhesion, it is important for test specimens to have both process conditions and film thickness that is representative of the target material system and interface. Sputtering, electroplating, electroless plating, evaporation, atomic layer deposition, etc. are some of the techniques used for depositing thin metal films, and the type of process chosen is dependent on the substrate on which deposition is done, the final thickness of the deposited metal, other materials in the specimen, etc. Thus, the resulting interfacial property is dependent on the process which is governed by, among other factors, the thickness of the film. The grain size, orientation, and how they grow from the substrate are also dependent mostly on the process parameters and on the final thickness of the film. In addition, when the film thickness is of the same order as the surface roughness of the substrate, the mechanical interlocking can play a more significant role in the measured interfacial fracture energy.

Relatively few studies have experimentally characterized interfacial FCP under constant stress amplitude loading, namely for metal-ceramic [1-7], ceramic-ceramic [8], polymer-metal [9-13], and polymer-glass [14] interfaces. Only a handful of these report delamination growth ratesfor systems involving thin films with thickness less than $10 \mu \mathrm{m}[1,5,7,8,10]$. This lack of available data is partly due to the reliance of these studies on sandwich-type test specimens, such as the double cantilever beam (DCB), double cleavage drilled compressive (DCBC), compact tension (CT), and four-point bend tests. The fabrication of these sandwich specimens, in which macroscale supporting substrates are bonded together by the thin films of interest, often involve unusual processes which result in non-representative films and interfaces. After bonding, specimens may then require precision cutting or drilling into the samples in order to initiate the crack, and/or have difficulty in controlling the crack propagation along the desired interface.

An alternative to the sandwich-style test is the peel test and its similar variations, which operate bystretching a partially detached film at an angle from the adhered substrate until the film delaminates. Although this test technique has been successfully used to characterize the critical interfacial fracture strength for many material systems, very few have reported testing films with thickness less than $10 \mu \mathrm{m}$ due to complications with specimen handling and applying 
peel forces through mechanical fixtures[15]. These peel tests are generally performed using displacement controlled actuators, which pull on the film at a constant rate while measuring the reaction force. Consequently, any FCP study to date that has utilized a peel-typetest has been conducted under constant displacement amplitude loading[16, 17].

To address the limitations of other test techniques, the Magnetically Actuated Peel Test (MAPT) was developed to characterize thin film interfacial delamination under load-controlled conditions. This technique has many advantages, including: (1) fixtureless experimental setup, (2) non-contact actuation, (3) simple batch fabrication with representative films and interfaces, (4) scalable from micron to submicron thick films, (5) ability to conduct interfacial FCP studies under constant stress amplitude loading, and (6) potential ability to conduct peel experiments while enclosing the specimen within a small environmental chamber. The MAPT technique has already been used previously to determine the critical interfacial fracture toughness of thin $\mathrm{Cu}$ films on Si substrates under monotonic loading [18]. The focus of this work is therefore to perform an interfacial FCP study using the MAPT technique, which to the authors' knowledge is a first for a peel-type testunder constant stress amplitude loading.

\section{Magnetically Actuated Peel Test Design}

In addition to the demonstrated ability to characterize a thin film interface under monotonic loading, the MAPT design is ideally suited to conduct interfacial FCP experiments under subcritical fatigue loading. As shown in Figure 1, the test specimen geometry features a patterned thin film such that three constant width strips connect to a circular central pad. A permanent magnet which is affixed onto the central pad is then is vertically displaced under the magnetic repulsive force from a nearby electromagnet. Thus, the remotely applied magnetic force is translated into peel forces along the three anchoring film strips. 


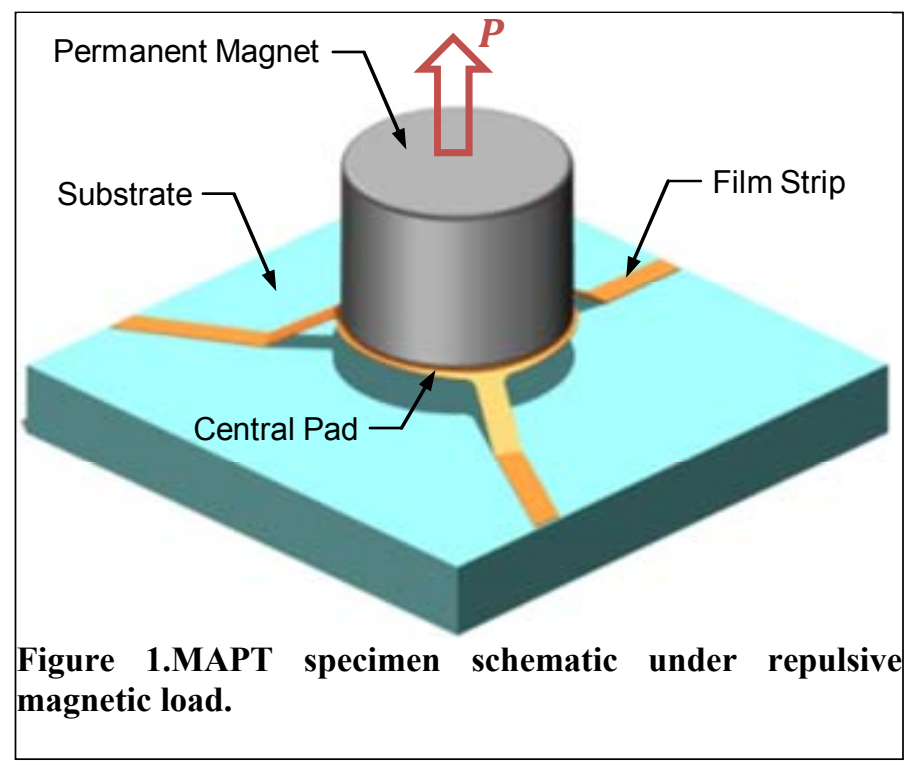

For monotonic fracture testing, the magnetic force is incrementally increased by supplying additional power to the driving electromagnet until the film strips delaminate from the substrate. A subcritical cyclic load can also be applied simply by supplying an AC current to the electromagnet. The amplitude and frequency of the applied force is thus a direct function of the input signal to the electromagnet. As the area above the specimen is free of obstruction, photographs of the test sample can be taken intermittently to capture the delamination propagation. Therefore, the crack growth rate can be observed as a function of the loading amplitude. In this work, a fatigue crack growth study was conducted for MAPT specimens featuring electroplated copper films on a silicon wafer under constant subcritical amplitude loading.

\section{Mechanics of Peeling}

The fatigue crack propagation rate is typically related the loading amplitude at the crack tip through the empirical Paris Law fit,

$$
\frac{d a}{d N}=\mathrm{C}(\Delta G)^{m}
$$


where $a$ is the crack length, $N$ is the number of loading cycles, $G$ is the Energy Release Rate (ERR), and $C$ and $m$ are constants determined through best fit with the experimental data. The ERR is classically defined through an energy balance as

$$
G=\frac{d}{d A}\left[W-U_{e}\right]
$$

where $A$ is the crack area, $W$ is the external work, and $U_{e}$ is the elastic strain energy. Most fracture tests therefore assume linear elastic conditions under which to evaluate the ERR. However, for ductile fracture, $G$ can seem relatively large due to the additional work required to offset the plastic dissipation in the system. In such cases, the global plastic work that is not inherent to the fracture process can be accounted for by using the Fracture Energy Rate (FER) parameter $\Gamma$ though

$$
\Gamma=\frac{d}{d A}\left[W-U_{e}-U_{p}\right]
$$

where $U_{p}$ is the plastic work. From an analytical and numerical modeling perspective, the external work and elastic strain energy are generally straightforward to calculate. The plastic dissipation, on the other hand, can be very challenging to accurately calculate when dealing with ductile materials.

As a simple first order peel test model shown in Figure 2a, Williams [19] assumes a flexible peel strip on a rigid substrate with uniform tensile strain in the released strip (i.e. ignoring bending), and develops an expression for the applied FER as

$$
\Gamma=\frac{F}{b}(1-\cos \theta+\varepsilon)-h \int_{0}^{\varepsilon} \sigma \cdot d \varepsilon,
$$

where $F$ is the peel force, $b$ is the strip width, $\theta$ is the peel angle, $\varepsilon$ is the tensile strain, $h$ is the strip thickness, and $\sigma$ is the tensile stress. 


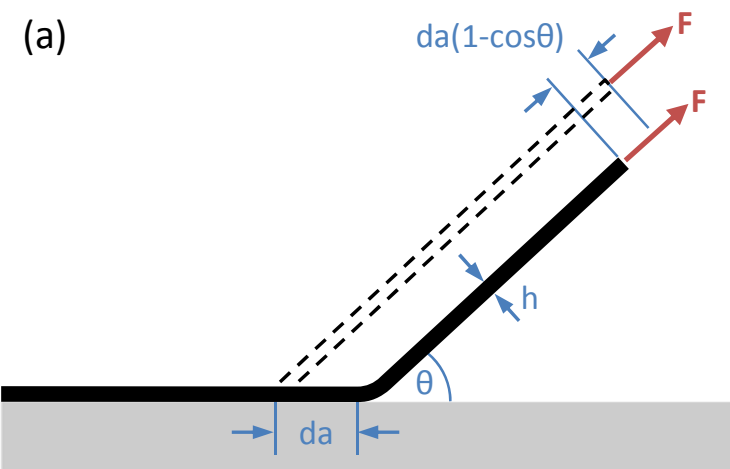

(b)

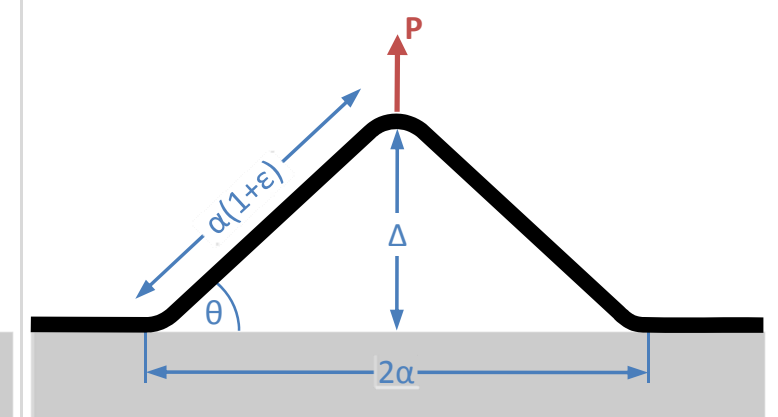

Figure 2.(a) Peel test as diagramed by Williams. (b) Pull-off test.

The MAPT design is analogous to the pull-off test shown in Figure 2b, where a vertical load $P$ applied at the center of a debonded strip imposes a simultaneous peel force on both adhered ends of the film. Equation (4) can thus be adapted to the MAPT design by incorporating three peel strips that are coupled to the central pad. Assuming symmetry and an initially flat and stress-free film, the following additional constraints can be imposed:

$$
\begin{aligned}
& \cos \theta=\frac{1}{1+\varepsilon}, \\
& P=3 F \sin \theta,
\end{aligned}
$$

where $P$ is the vertical magnetic force. Substituting (5) into (4) and solving for $\Gamma / h$ results in

$$
\frac{\Gamma}{h}=\sigma \frac{\varepsilon(2+\varepsilon)}{1+\varepsilon}-\int_{0}^{\varepsilon} \sigma \cdot d \varepsilon .
$$

Equation (7) shows the ratio of FER to film thickness as a function of tensile stress and strain. Alternatively, the ERR can similarly be calculated by incorporating only the elastic strain energy which results in

$$
\frac{G}{h}=\sigma \frac{\varepsilon(2+\varepsilon)}{1+\varepsilon}-\frac{\sigma^{2}}{2 E}
$$

where $E$ is the elastic modulus of the film. Additionally, (5) and (6) can be combined into

$$
\frac{P}{b h}=3 \sigma \frac{\sqrt{\varepsilon(2+\varepsilon)}}{1+\varepsilon}
$$


Since Equations (7-9) are in terms of only stress and strain, we can implicitly solve for $G$ or $\Gamma$ in terms of the magnetic force and cross section of the film strip for a given film constitutive model. It should be noted that the film material is not necessarily limited to an elastic analysis, since a nonlinear stress-strain relation can be used to model the elastoplastic stretching of the adherend. A nonlinear model for $\mathrm{Cu}$ thin films derived from [20]was used for calculations, which can be approximated with the elastic-plastic power law hardening constants $E=110 \mathrm{GPa}$, $K=1490 \mathrm{MPa}$, and $n=0.24$, where $K$ and $n$ are the hardening modulus and hardening exponent, respectively. The ERR and FER are compared as a function of the magnetic force in Figure 3. The two curves are observed to diverge meaningfullyfor $P / b h>75 \mu \mathrm{N} / \mu \mathrm{m}^{2}$, which isbeyond the force level applied to MAPT specimens in this work. Therefore, it is sufficient in this case to use the loading amplitude $\Delta G$ for characterization of the interfacial delamination between the peeling film and substrate.Additionally, the length of the peeling strip does not affect the calculations. Therefore, $\Delta G$ is only dependent on the magnetic load and is stable as the delamination progresses.

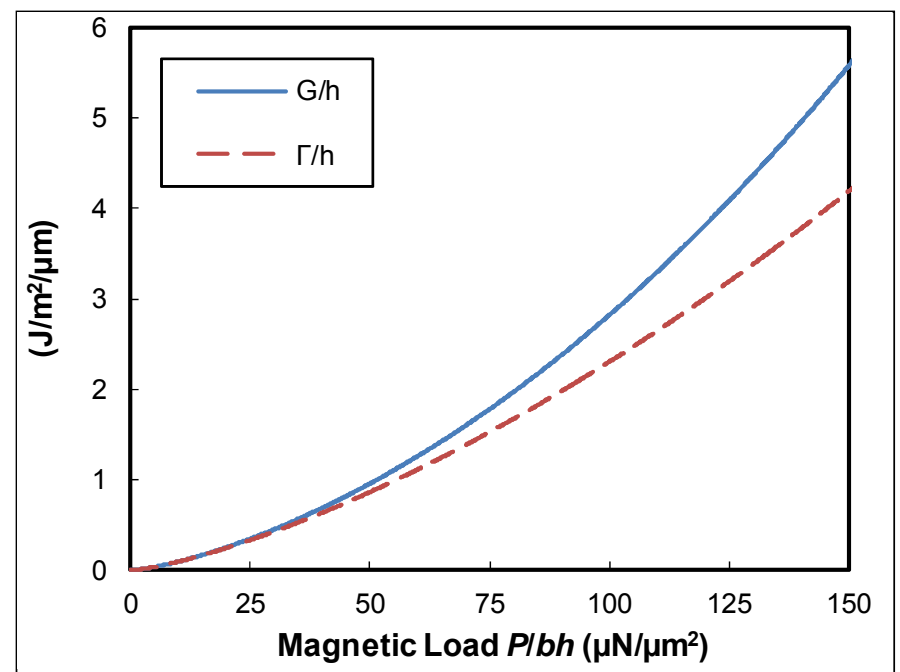

Figure 3. Comparison of ERR and FER as a function of the applied magnetic load.

The equations derived above apply for the case that the peeling films are initially flat and stress-free. However, during sample fabrication the released film strips of a MAPT specimen may not lie perfectly flat on the substrate, but instead have some residual slack after removal of 
the underlying sacrificial layer. This residual deformation can be accounted for in the analysis by modifying (5) such that

$$
\cos \theta=\frac{1}{(1+\varepsilon)\left(1+\varepsilon_{0}\right)}
$$

where $\varepsilon_{0}$ is a constant valuerepresenting the initial slack in the film but does not contribute to the mechanical strain. If there is no initial slack (i.e. $\varepsilon_{0}=0$ ), then (10) simply reduces to (5). Consequently, Equations (7) and (9) can be updated to include the slack term such that,

$$
\frac{\Gamma}{h}=\sigma\left(1-\frac{1}{(1+\varepsilon)\left(1+\varepsilon_{0}\right)}+\varepsilon\right)-\int_{0}^{\varepsilon} \sigma \cdot d \varepsilon,
$$

and

$$
\frac{P}{b h}=3 \sigma \sqrt{1-\frac{1}{\left((1+\varepsilon)\left(1+\varepsilon_{0}\right)\right)^{2}}}
$$

Assuming the same nonlinear constitutive model for $\mathrm{Cu}$ thin film,the impact of slack on the resulting peel angle is shown inFigure 4. For applied force values $\mathrm{P} / \mathrm{bh}<150 \mu \mathrm{N} / \mu \mathrm{m} 2$, it is shown that even a relatively small amount of slack can increase the resulting peel angle when loaded by a few degrees.

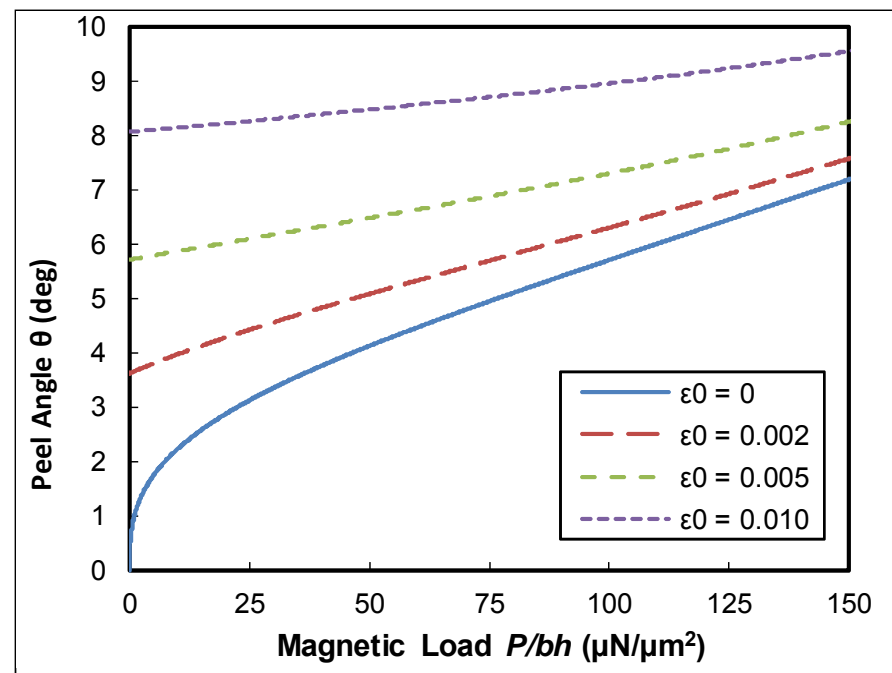

Figure 4.Effect of initial slack in the peeling strips on the peel angle. 
Even though the peel angle may be sensitive to initial slack, the resulting calculation for the FER is quite insensitive as shown inFigure 5. There is virtually no effect on the calculated FER for $\varepsilon_{0}=0.2 \%$ despite the increased peel angle. As will be discussed in detail in a future paper, the amount of slack in the MAPT specimens in this work was measured to be no more than $0.3 \%$, and therefore did not factor into the calculations for either $G$ or $\Gamma$.

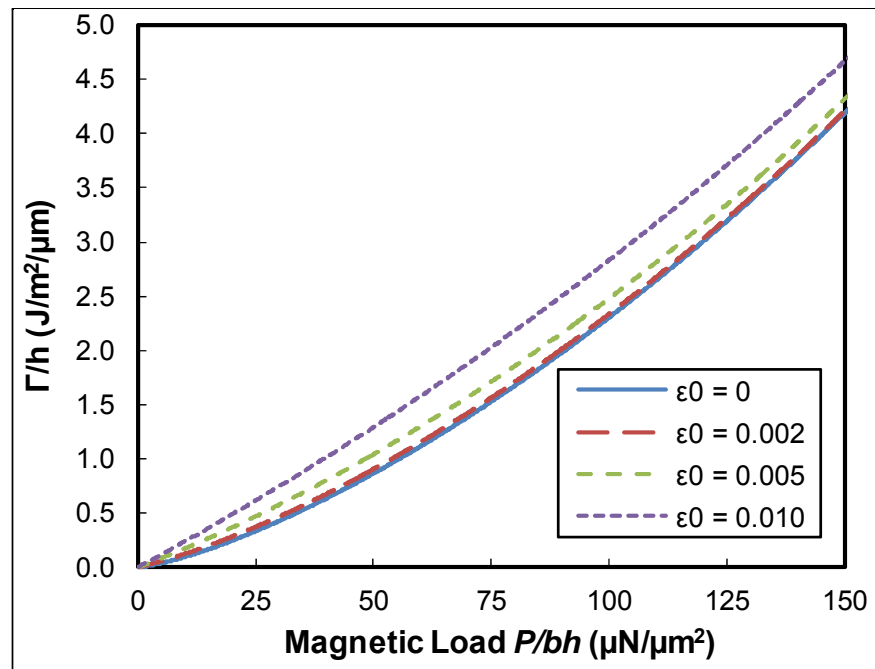

Figure 5.Effect of initial slack in the peeling strips on the applied FER.

\section{SAMPle Preparation}

Test specimen fabrication details are presented elsewhere $[18,21]$ and are summarized here. Copper seed layer was first sputtered $500 \mathrm{~nm}$ thick onto a bare silicon wafer and then electroplated an additional $1.1 \mu \mathrm{m}$ for a total film thickness $h$ of $1.6 \mu \mathrm{m}$. Depending on the mask design, the film strips have constant width $b$ of 75-200 $\mu \mathrm{m}$, and initially released length $\alpha$ of 250$1000 \mu \mathrm{m}$. A thin sacrificial polymer disc between the film and substrate was removed in order to release the central pad. A Ni-plated neodymium magnetic cylinder (1/16" x 1/16") was then assembled onto the central pad and adhered using a surface mount epoxy.

A completed MAPT specimen is shown in the unloaded and loaded state inFigure 6. The permanent magnet assembly process was developed for accurate placement with strong and uniform adhesion to the central pad. The resulting vertical translation of the magnet is stable 
under load and promotes even stretching of the three anchoring peel strips. Visible hash marks which run alongside the peel strips were also incorporated into the mask design, and are a convenient reference to determine the delamination front position through visual inspection.
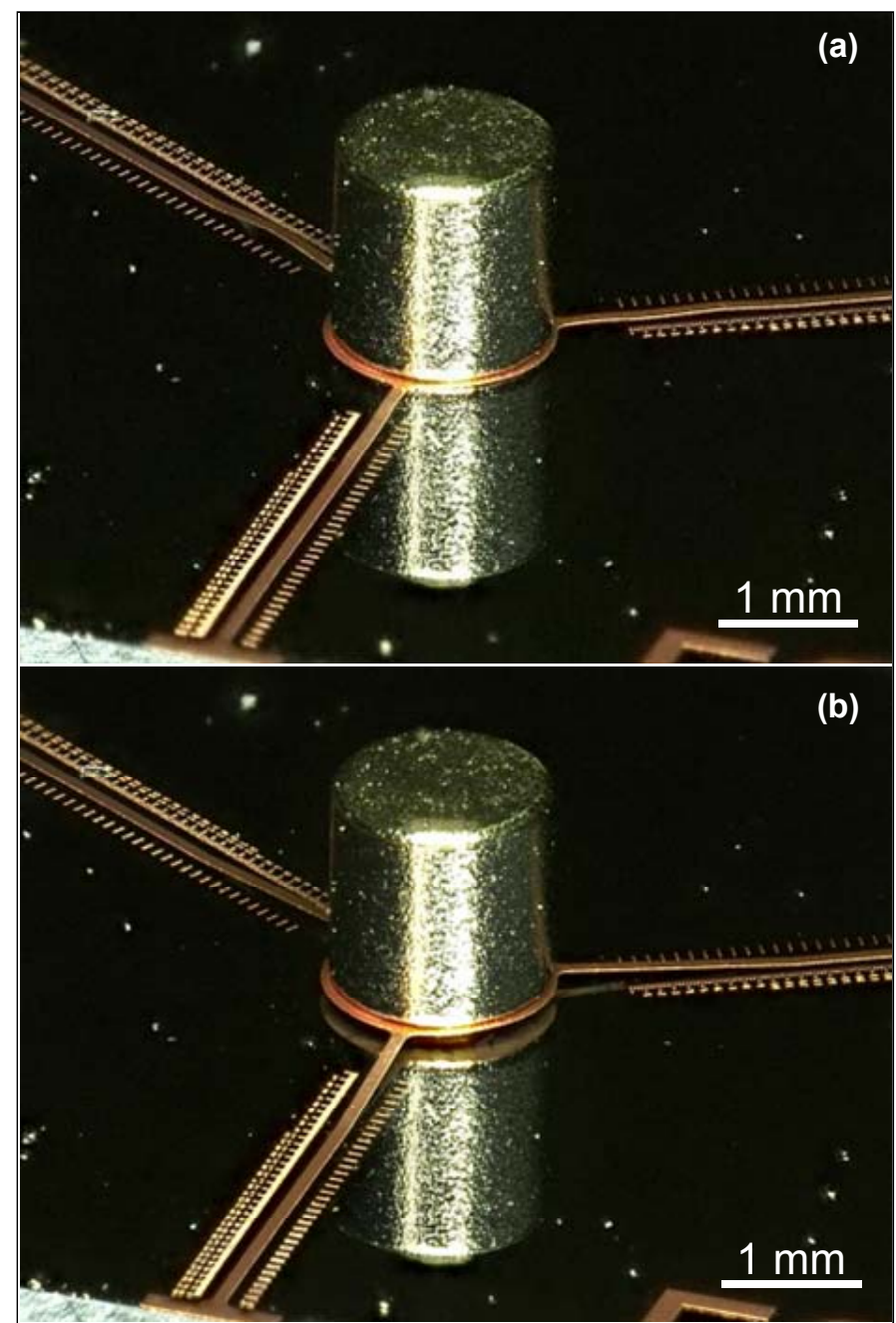

Figure 6. MAPT specimen (a) at rest with no applied force and (b) with permanent magnet elevated due to repulsion from driving electromagnet below, and imposing peel forces along the three anchoring strips.

\section{EXPERIMENTALDESIGN}

\subsection{Setup}

The experimental setup for MAPT specimens subjected to cyclic fatigue loading is shown in Figure 7. The test specimen is first placed onto a stand, and then moved into alignment with 
the electromagnet below. The outer edges of the specimen substrate are taped to the stand to keep itstationary during loading. An optical microscope is positioned above the test specimen, and was used to capture the cyclic displacement and delamination propagation of the film strips. All objects in the near vicinity of the test specimen, such as the stand and microscope, do not contain magnetic materials and thus did not affect the applied magnetic force.

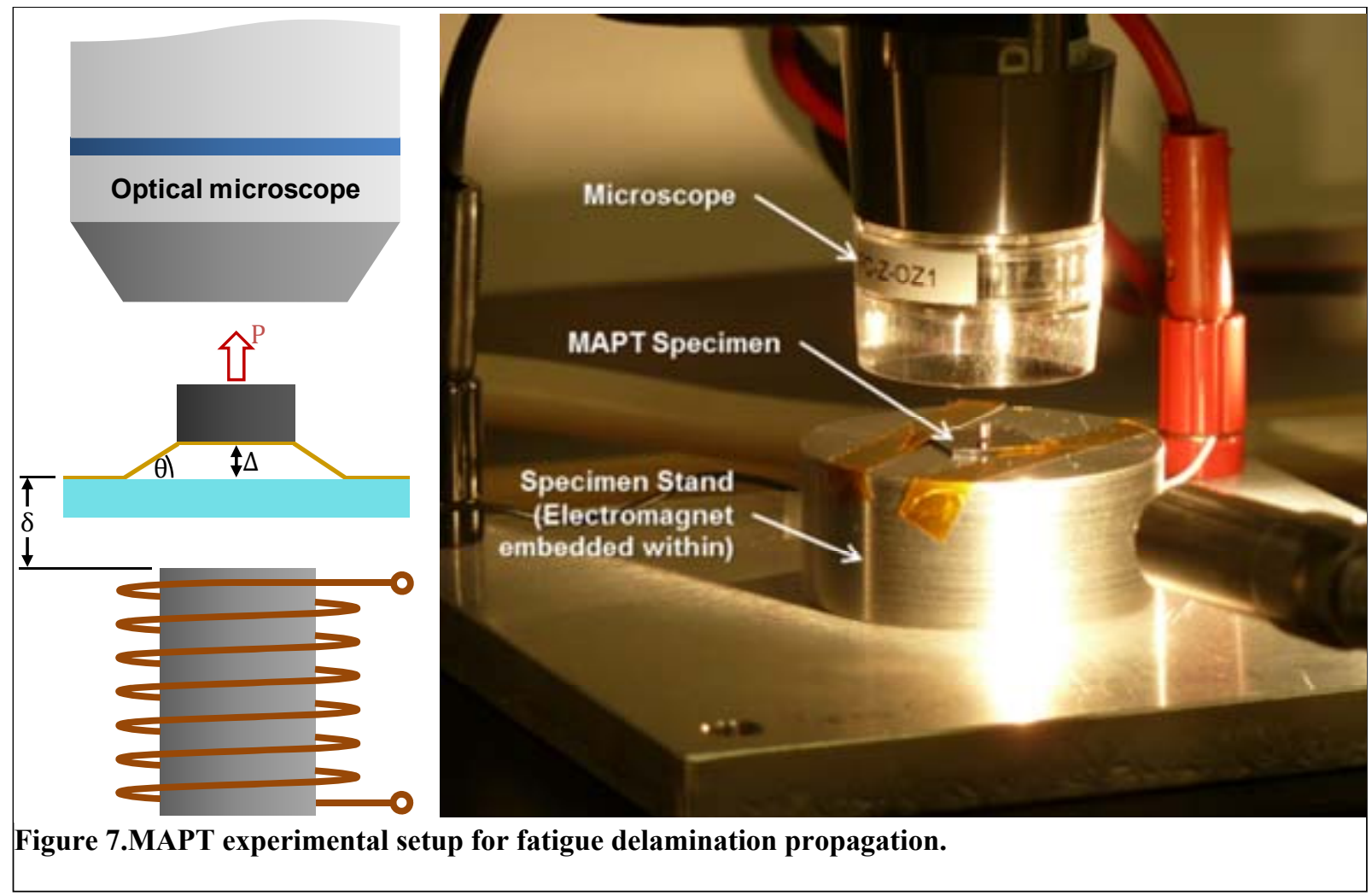

\subsection{Application of Cyclic Loading}

In order to minimize the dynamic kinetic effects of cyclic loading, the electromagnet was driven by a voltage sine wave from $V_{\min }$ to $V_{\max }$ at $500 \mathrm{mHz}$; a frequency far below the resonantfrequency of the test samples, yet fast enough to apply several thousand cycles per hour.

It is well known that the loading ratio $R$ can significantly affect the crack growth rate. Since the MAPT design does not allow for negative vertical displacement (i.e. the released film structure cannot penetrate the substrate), the available range of stress ratios are $R \geq 0$. In this work, crack propagation was studied under $R=0$ fatigue loading by ensuring the permanent 
magnet and attached central pad make contact with the substrate at the bottom of the loading cycle (i.e. at $\left.V_{\min }\right)$.

A force calibration was performed under monotonic loading conditions as reported in [18], which resulted in positive repulsive force when the voltage applied to the electromagnet was greater than $2.7 \mathrm{~V}$ for a separation gap $\delta$ of $1.8 \mathrm{~mm}$. However, a separate force calibration under cyclic voltage loading was not feasibledue to limitations of the described setup. Therefore, the magnetic field strength (and thus the total magnetic force) generated by the driving electromagnet when the voltage reaches $V_{\max }$ during cycling is assumed to be equivalent to the case where the voltage is held constant at $V_{\max }$. The voltage was thus cycled from $1.8 \mathrm{~V}$ to $V_{\max }$ to ensure $R=0$ loading, where the maximum force at $V_{\max }$ was derived from the monotonic calibration results. Fatigue cycling tests were conducted for loading amplitudes $\Delta P / b h$ ranging from 31.8 to $43.8 \mu \mathrm{N} / \mu \mathrm{m}^{2}$.

\subsection{Procedure}

Fatigue testing was performed in a laboratory environment at $22^{\circ} \mathrm{C}$ and $50 \%$ relative humidity. MAPT specimens were subjected to a series of constant amplitude loading blocks of $N$ $\approx 15,000-125,000$ cycles each, depending on the resulting delamination rate. Since the length of peeling per strip during one loading block was about 0 to $1,000 \mu \mathrm{m}$, a total of about five individual loading blocks could be applied to one specimen before the entire 4,000 $\mu \mathrm{m}$ long peel strip was consumed. The rate of delamination propagation $d a / d N$ within each loading block was captured using time-lapse photography, where the optical microscope captured photographs of

the specimen intermittently every few minutes. With proper in-plane lighting, the location of the peeling edge of each strip was determined from these images as shown in Figure 8, and thus provided a means to monitor the crack propagation over time. 


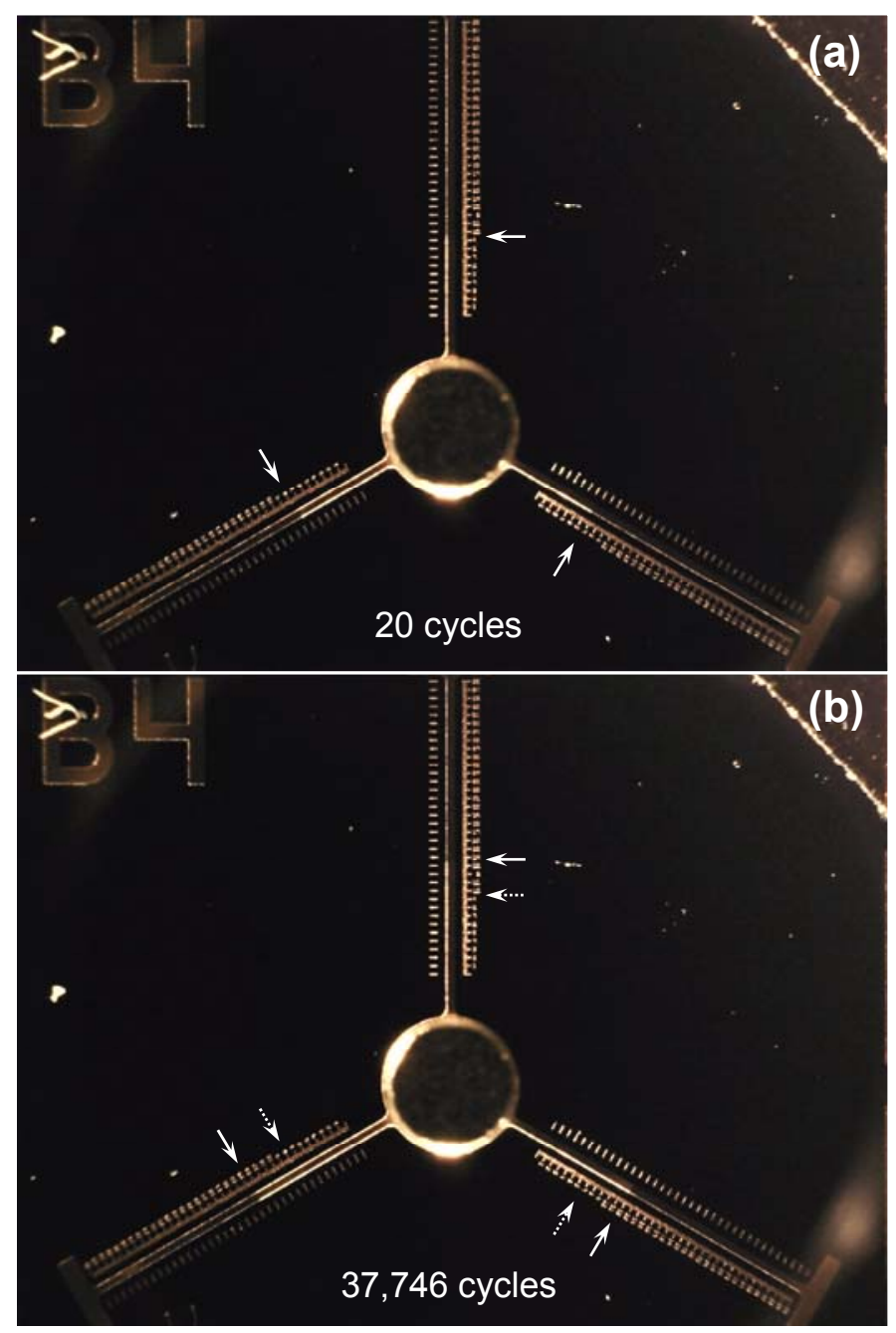

Figure 8.Top-down view of MAPT specimen undergoing fatigue cycling from 1.80 - $6.99 \mathrm{~V}$ after (a) 20 cycles and (b) 37,746 cycles. Solid arrows indicate current delamination front position, with dashed arrows in (b) indicating the delamination position at the start of fatigue cycling.

\section{Results}

The delamination of each strip was tracked individually, and typical delamination progression results are shown in Figure 9 over a range of loading amplitudes. No crack propagation was observed for $\Delta P / b h \leq 31.8 \mu \mathrm{N} / \mu \mathrm{m}^{2}\left(\Delta G \leq 0.78 \mathrm{~J} / \mathrm{m}^{2}\right)$ for as long as 125,000 cycles, so this is considered to be roughly the endurance limit. While some variability in peeling behavior amongst the strips was observed for lower loading amplitudes $\left(\Delta P / b h<37 \mu \mathrm{N} / \mu \mathrm{m}^{2}\right)$, the 
delamination rate for the three strips was observed to be typically more consistent and uniform for larger loading amplitudes.
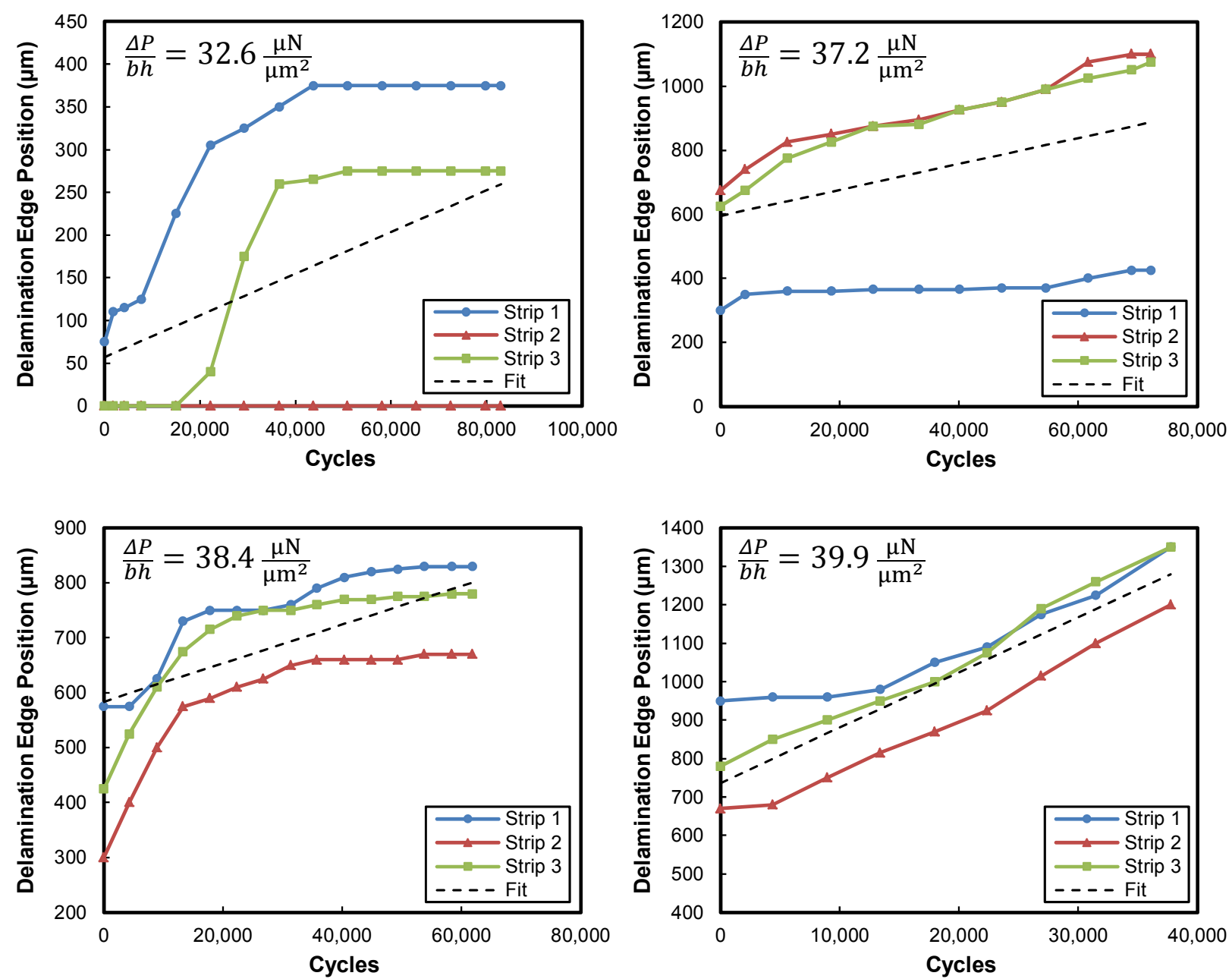

Figure 9. Delamination propagation at various loading amplitudes $(R=0)$. The dashed line represents the average linear fit amongst the three strips for a given loading block.

An average delamination rate within each loading block was determined from the slope of a linear fit of the cumulative crack propagation data. This averaged crack growth rate is shown in log-scale relative to the applied $\Delta G$ in Figure 10. A Paris Law fit of the delamination rates results in constants $\mathrm{C} \approx 3 \cdot 10^{-9}$ and $\mathrm{m} \approx 15$, and has good correlation with the experimental data. Monotonic testing results for identical samples as discussed in [18] are also shown and indicate the range of critical loading amplitudes for steady-state peeling. 


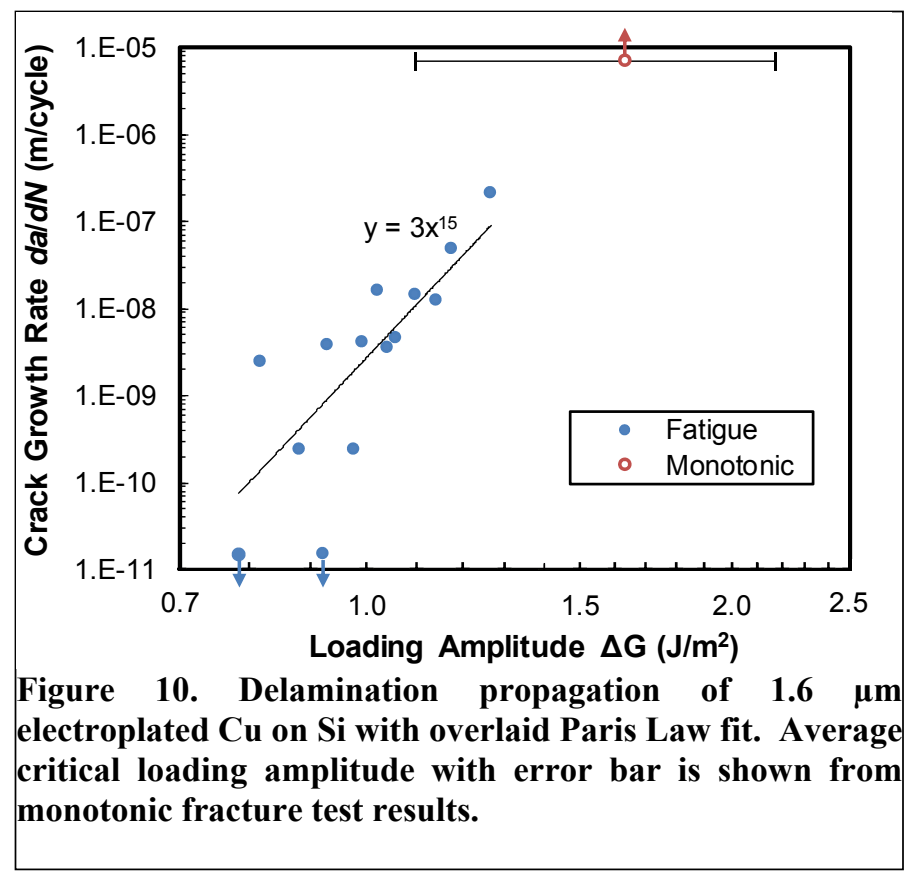

\section{DISCUSSION}

The overall trend observed for delamination growth rate is consistent with typical fatigue crack behavior; a threshold level for crack advance followed by a Paris regimefor propagation and finally unstable growth near the critical limit. Since a sharp crack tip is inherently present between the peel strip and substrate upon fabrication of the test specimen, the resulting crack propagation from cyclic subcritical loading is considered Stage-II crack growth.

For larger loading amplitudes, each of the three strips behaved similarly with relatively constant delamination growth rates. However, at lesser amplitudes, there is increased scatter in the crack growth rate, with appreciable deviation in fatigue behavior between the three strips. In such cases, the delamination progressed with varying speed, often with one or more strips experiencing temporary crack arrest or not delaminating at all. This pronounced variability under low amplitude fatigue loading may be attributable to the thin membrane effect, where the out-of-plane deformation is very sensitive to small forces until the film is sufficiently stretched. Additionally, there may be inherent variability due to the scale effect in thin film peeling, as discontinuous peeling behavior has been reported for $<10 \mu \mathrm{m}$ thick copper films $[15,22]$. 


\section{CONCLUding Remarks}

To the authors'knowledge, this work is the first to report delamination propagation rate as a function of constant amplitude fatigue loading using a peel test configuration. For $1.6 \mu \mathrm{m}$ thick $\mathrm{Cu}$ films on a Si substrate, interfacial fatigue crack propagation was observed for a minimum applied ERR amplitude that was about half the critical value required for steady-state peeling. Intermediate loading amplitudes resulted in a Paris Law regime with relatively predictable delamination rates.

The focus of this work is in development and demonstration of an experimental procedure for characterizing thin film delamination under both monotonic and fatigue loading. Thus, further investigation into more sophisticated analytical and finite element models of the peeling mechanics, beyond the uniform tension assumption described in this work, will be discussed in a future publication. In addition, since no physical contact with the test specimen is required for loading the interface, MAPT specimens can be housed within a small enclosed chamber for testing under high temperature/high humidity conditions, as is part of ongoing research.

\section{ACKNOWLEDGMENTS}

This work is supported by the National Science Foundation (NSF) under grant No. CMMI0800037 .

\section{REFERENCES}

[1] R. M. Cannon, B. J. Dalgleish, R. H. Dauskardt, T. S. Oh, and R. O. Ritchie, "Cyclic fatigue-crack propagation along ceramic/metal interfaces," Acta Metallurgica et Materialia, vol. 39, p. 2145, 1991.

[2] M. C. Shaw, D. B. Marshall, B. J. Dalgleish, M. S. Dadkhah, M. Y. He, and A. G. Evans, "Fatigue crack growth and stress redistribution at interfaces," Acta Metallurgica et Materialia, vol. 42, p. 4091, 1994.

[3] J. M. McNaney, R. M. Cannon, and R. O. Ritchie, "Fracture and fatigue-crack growth along aluminum-alumina interfaces," Acta Materialia, vol. 44, p. 4713, 1996.

[4] J. J. Kruzic, R. A. Marks, M. Yoshiya, A. M. Glaeser, R. M. Cannon, and R. O. Ritchie, "Fracture and fatigue behavior at ambient and elevated temperatures of alumina bonded 
with copper/niobium/copper interlayers," Journal of the American Ceramic Society, vol. 85, pp. 2531-2541, Oct 2002.

[5] J. J. Kruzic, J. M. McNaney, R. M. Cannon, and R. O. Ritchie, "Effects of plastic constraint on the cyclic and static fatigue behavior of metal/ceramic layered structures," Mechanics of Materials, vol. 36, pp. 57-72, Jan-Feb 2004.

[6] M. Hasegawa and Y. Kagawa, "Decohesion behavior in copper-sapphire interface under mode I cyclic loading," Materials Science and Engineering a-Structural Materials Properties Microstructure and Processing, vol. 417, pp. 158-165, Feb 152006.

[7] H. Hirakata, M. Kitazawa, and T. Kitamura, "Fatigue crack growth along interface between metal and ceramics submicron-thick films in inert environment," Acta Materialia, vol. 54, pp. 89-97, Jan 2006.

[8] Q. Ma, "A four-point bending technique for studying subcritical crack growth in thin films and at interfaces," Journal of Materials Research, vol. 12, pp. 840-845, Mar 1997.

[9] J. Guzek, H. Azimi, and S. Suresh, "Fatigue crack propagation along polymer-metal interfaces in microelectronic packages," IEEE Transactions on Components, Packaging, and Manufacturing Technology Part A, vol. 20, p. 496, 1997.

[10] W. Xie and S. K. Sitaraman, "Investigation of interfacial delamination of a copper-epoxy interface under monotonic and cyclic loading: Experimental characterization," IEEE Transactions on Advanced Packaging, vol. 26, pp. 447-452, 2003.

[11] M. Dessureault and J. K. Spelt, "Observations of fatigue crack initiation and propagation in an epoxy adhesive," International Journal of Adhesion and Adhesives, vol. 17, p. 183, 1997.

[12] D. Samet, A. Kwatra, and S. K. Sitaraman, "A Compliance-Based Approach to Study Fatigue Crack Propagation for a Copper-Epoxy Interface," in ASME 2015 International Technical Conference and Exhibition on Packaging and Integration of Electronic and Photonic Microsystems collocated with 13th International Conference on Nanochannels, Microchannels, and Minichannels, San Francisco, CA, 2015.

[13] D. Samet, A. Kwatra, and S. K. Sitaraman, "Cohesive Zone Parameters for a Cyclically Loaded Copper-Epoxy Interface," in Electronic Components and Technology Conference (ECTC), 2016 IEEE 66th, Las Vegas, NV, 2016.

[14] J. E. Ritter, W. Grayeski, and T. J. Lardner, "Cyclic fatigue - Crack growth along polymer/glass interfaces," Polymer Engineering and Science, vol. 36, pp. 2382-2388, Sep 1996.

[15] H. F. Zhao and Y. G. Wei, "Determination of interface properties between micron-thick metal film and ceramic substrate using peel test," International Journal of Fracture, vol. 144, pp. 103-112, Mar 2007.

[16] S. Sohn, "A new method based on application of cyclic strain to evaluate the durability of pressure sensitive adhesives," Journal of Adhesion Science and Technology, vol. 17, pp. 1039-1053, 2003.

[17] K. Liao and K. T. Wan, "Delamination behavior of film-substrate systems under cyclic loading," Journal of Materials Science Letters, vol. 19, pp. 57-59, Jan 2000.

[18] G. T. Ostrowicki and S. K. Sitaraman, "Magnetically actuated peel test for thin films," Thin Solid Films, vol. 520, pp. 3987-3993, 2012.

[19] J. G. Williams, "Energy release rates for the peeling of flexible membranes and the analysis of blister tests," International Journal of Fracture, vol. 87, pp. 265-288, 1997. 
[20] C. A. O. Henning, F. W. Boswell, and J. M. Corbett, "Mechanical properties of vacuumdeposited metal films - I. Copper films," Acta Metallurgica, vol. 23, pp. 177-185, 1975.

[21] G. T. Ostrowicki, N. T. Fritz, R. I. Okereke, P. A. Kohl, and S. K. Sitaraman, "Domed and released thin-film construct - An approach for material characterization and compliant interconnects," IEEE Transactions on Device and Materials Reliability, vol. 12, pp. 15-23, Mar 2012.

[22] K. S. Kim and J. Kim, "Elasto-plastic analysis of the peel test for thin-film adhesion," Journal of Engineering Materials and Technology, vol. 110, pp. 266-273, Jul 1988. 